\title{
Exploration of How Female Body Image Is Presented and Interpreted on Instagram
}

\author{
Yang Hu \\ Department of Communication and Media, School of Arts, University of Liverpool, Liverpool, England \\ Email:1448166215@qq.com
}

How to cite this paper: $\mathrm{Hu}, \mathrm{Y}$. (2018). Exploration of How Female Body Image Is Presented and Interpreted on Instagram. Advances in Journalism and Communication, 6, 95-120. https://doi.org/10.4236/ajc.2018.64009

Received: September 26, 2018

Accepted: December 2, 2018

Published: December 5, 2018

Copyright (C) 2018 by author and Scientific Research Publishing Inc. This work is licensed under the Creative Commons Attribution International License (CC BY 4.0).

http://creativecommons.org/licenses/by/4.0/

\section{cc) (7) Open Access}

\begin{abstract}
The aim of the research was to explore the ways that female body image was presented and interpreted on Instagram. Specifically, it aimed to identify how women disseminate their body image on Instagram, examine how viewers respond to women's body image disseminated on Instagram and determine how the women's body image presentation on Instagram influences the viewers' self-perceptions and behavior. To achieve the goal, content analysis was used. The data set consisted of 140 photographs that were collected from hashtags. It was found that body images presented in Instagram highlighted sexuality and attractiveness by emphasizing slim body, long hair, with fewer clothing, followed by photographs communicating business purposes and communicating the ideal body, but only a few photos challenged the majority by creating totally different images, such as women's lifting heavy weights or doing something adventure and wearing a bikini at a larger size. As for the viewers' responses, it was found that most viewers positively respond to body image photographs in Instagram, appreciating wonderful and ideal body image and admiring the transformation. In addition, it was found that many comments show that the viewers are dissatisfied with themselves after comparing their body images with ideal body images. This further suggested self-objectification. As for influence, it was found that the women's body images communicated on Instagram influence the viewers significantly in the following aspects: 1) arousing consciousness and inspiration so as to encourage them to make changes; 2) triggering self-objectification; 3 ) inspiring use of a new perspective to perceive and expect the body image. The research, nevertheless, was limited in sampling which reduced generalizability of findings.
\end{abstract}

\section{Keywords}

Women's Body Image, Instagram, Presentation, Influence 


\section{Introduction}

\subsection{Research Background}

Numerous accredited scholars, like Coppock et al. (2014), Tiidenberg and Gómez-Cruz (2015) and Lee et al. (2014) are studying and evaluating the influence of mass media on body images of women. Technology has played a part in influencing women's body images. Technology and social sites are playing a major role in redefining beauty standards for females (Andsager, 2014: p. 407). For instance, Instagram is known as one of the widely used space or social sites that influence perceptions of people about the ways in which women should maintain their body image (Halliwell, 2015: p. 177). Recent developments in the usage of social sites, like Instagram, have further constructed new and different ideas and expectations of society about the ways in which body of women should appear (Andsager, 2014: p. 407). However, the general view of body of knowledge of this field depicts that focus of body image research has drastically changed over decades. In fact, researchers such as Lewallen and Behm-Morawitz (2016) have identified and concluded that individuals in society have become significantly concerned about their body images. In particular, despite researches have made attempt to explore influence of social media on women's body image, little understanding has been obtained in terms of what kinds of images women display on Instagram. In addition, there is limited knowledge of how the viewers respond to the body images displayed on Instagram.

\subsection{Research Rationale}

Instagram is considered one of the most popular online photo-sharing social media apps, and an effective approach to disseminating information about body images (Hempel, 2014: p. 73). In other words, it has become an important online space where women share body images and negotiate their own identity around their body. A distinct feature of Instagram lies in "fitspiration" images (a term combing fitness and inspiration). There is a trend of women to display images so as to encourage others to exercise and have a healthy diet (Tiggemann \& Zaccardo, 2015). Previous studies have demonstrated the connection between women's body image and social media, such as Facebook (Fardouly \& Vartanian, 2016; Mabe et al., 2014), but few efforts have been made to explore how Instagram acts a platform for women to disseminating their body image. Practically, the author intends to study female body image representation on Instagram with the aim of critically evaluating images available to women with the aim of ensuring there is awareness of potential limited nature of healthy body images available.

\subsection{Research Aim and Objectives}

The present study aspires to explore and evaluate the ways in which female body image is presented and interpreted on Instagram. The study has further exam- 
ined women's body presented on social media. To achieve the stated aim, author has developed following specific objectives:

- To identify how women disseminate their body image on Instagram;

- To examine how viewers respond to women's body image disseminated on Instagram;

- To determine how the women's body image presentation on Instagram influences the viewers' self-perceptions and behavior.

\section{Literature Review}

\subsection{Introduction}

The literature review section of the dissertation facilitates in reviewing available information about the subject. Apparently, the discussion in this section attempts to summarize past arguments of accredited scholars about women's body image and feminism. In particular, the study emphasize on explaining the key variables of the study specifically in accordance with available information and data. Substantially, the chapter focuses on interpreting theoretical dimensions of the research. To be specific, the discussion is accountable of defining feminism, women's body and post-feminism. The discussion is comprised of subjective information which is extracted from secondary sources like past studies and publications.

\subsection{Feminism and Women's Body: Feminism vs. Post-Feminism}

The term feminism can be narrowly defined as the advocacy of women's rights based on the sexes equality (Peterson et al., 2008). Broadly speaking, it can be understood asa range of ideologies, political movements and social concerns that share particular and common goals. In certain, it attempts to define, explain, establish and acquire economic, personal, political and social equality of sexes. In particular, the term further refers to the advocacy of women's rights on the basis of equality of genders (Brown, 2017). Fardouly and Vartanian (2016) explained that feminism is a concept that emphasizes on explaining women's rights and interests. In addition, it is a movement that seeks superior rights based on the equality of sexes. It attempts to evaluate overall privilege for women throughout the process of fighting for their basic rights. In similar manner, Banet-Weiser (2018) stated that post-feminism is another important term being used in social science fields. The concept of post-feminism was developed after 1960s and it has re-established in late 1980s. In accordance to Feltman and Szymanski (2018), as an ideology, the concept of post-feminism was defined in accordance with ideology which depicts that women have acquired relative equality. However, women still want more which further depicts that women could use their specific sexuality for the purpose of acquiring empowerment.

In addition, Fardouly et al. (2015) noted that feminism theory is focused on redefining the ways in which society trends are developed for women. The feminism discourse refers to the development of complex dynamics that exists 
among ideologies, languages and power. Practically, the theory of feminism in terms of materialistic ideologies is accountable of forming feminism perceptions. For instance, Fardouly, Willburger and Vartanian (2018) cited that body images are regarded as the source of materialistic ideologies and assumptions. These ideologies are said to be the key factors affecting ideological relationship to women. In addition, the study of Banet-Weiser (2018) explained that socialist feminism facilitates in distinguishing liberation of women. In general, development of unrealistic body images and standards for women is also regarded as discrimination. Many feminist organizations have initiated movements that are simply against patriarchy. The study of Slater, Varsani and Diedrichs (2017) also supported the fact that feminism is further effective in eliminating the requirements and unrealistic standards of social positions.

In particular the standpoint theory can be viewed as an important feminist theoretical point of view. Tiggemann and Zaccardo (2015); Modleski (2014) stated that standpoint theory suggests that social positions of individuals are based on different perspectives. These perspectives tend to argue about the theories that treat women in an uncertain form. Arguably, feminism tends to eliminate socio-cultural standards of feminine beauty that is simply presented in almost different forms of popular media projections. The study of Cameron, Ward, Mandville-Anstey and Coombs (2018) explained that ideal body discrimination can cause standard issues and problems for people. It creates discrimination. The idea of post feminism has also become a significantly contested term within lexicon of feminist culture. Moreover, in recent era, several scholars and practitioners are found to be debating about the feminism and gender consciousness of young women. In accordance to Tiggemann and Zaccardo (2015); Modleski (2014), in relation to post feminism, the body image of women is found to be overloaded with assumptions that indicate the existence of assumptions that are worth struggling over. In recent past decades, much of the arguments about post feminism are found to be associated with the development of mutual relationship.

Post-feminism and media culture are regarded as critical objects that scholars and investigators of culture should inquire and study. In particular, the notions and ideas in this regard suggest that one of the striking aspects of post feminist media culture is explained as its pre-occupation with body. Post feminism idea depicts that femininity is substantially defined as a bodily property rather than a social construct. For instance, Salter (2016); Bruce (2016); McLean, Paxton and Wertheim (2016) explained that instead of adoring motherhood for females, the societal norms are engaging women in the race of maintaining toned bodies. Conceptually, femininity is explained via unrealistic body images equipped with glamour and glorifying details instead of nurturing or motherhood. As stated by Toffoletti and Thorpe (2018), body is determined to be presented simultaneously as the source of power and attractiveness. These defined beauty standards for women are causing certain challenges and problems. 


\subsection{Feminism and Women's Body Image in the Media}

According to Tiggemann and Zaccardo (2015); Modleski (2014), media's portrayal of women should focus on spreading awareness about feminism, that is, media should report in a way for respecting the women and advocating sex equalities. However, in this era, media is widely used for redefining and remodeling the beauty standards of femininity. Feminism in media is wrongly portrayed which can affect the overall self-esteem and perception of young females. Arguably, Ceulemans and Fauconnier (2015) explained that feminism should be focused on eliminating false standards and unrealistic requirements of society for young female's bodies. In spite of eliminating false standards and perceptions, media is actively participating in narrowing down the body requirements of females. These social standards and requirements are imposing negative influences for women. The study by Coppock, Haydon and Richter (2014) explained that body image can be simply defined as an individual's perception about own body structure, weight and skin tone. The overall process of developing body image can influence overall perceptions and assumptions about the considerable standards of gender differences. In fact, the development of media across the globe has transformed the way people perceive interactions and objectification.

The rise in media across the globe has significantly contributed towards the development of women objectification and body image development (Kinsaul, Curtin, Bazzini, \& Martz, 2014; Weidhase, 2015). From a feministic view point, body image of women is perceived as ideal in its real condition. The integration of unhealthy methods to become skinny is against feminism policy because it tends to avoid the objectification of women in society. Moreover, the feministic approaches can challenge unrealistic perceptions and social requirements so as to ensure that women have access to equal rights (Andsager, 2014). Feminism and body image are constructed on the concept of self-branding which is something known as important for the development of distinctive public images (Marwick, 2010). In specific, decades of investigation and research about the feminism and women's body image portrayal in media has documented considerable amount of negative impacts and influences (Kinsaul, Curtin, Bazzini, \& Martz, 2014; Weidhase, 2015). Traditionally, media images portray women in a way in line with male's expectations such as sexuality. These unrealistic expectations of society create significant hurdles for women (Grogan, 2016). In addition, the arguments of Tiidenberg and Gómez-Cruz (2015) explained that feminism approaches are effective in formulating standard assumptions associated with media portrayal and demonstration. It tends to promote the idea of becoming socially acceptable and recognized. Moreover, feministic approaches must emphasize on creating strong perception of people about women and their rights. These approaches are further accountable of struggling against women objectification in media. The study of Retallack, Ringrose and Lawrence (2016) explained that the feministic movements must focus on eliminating women's 
body concerns because it possesses significantly negative influence for them.

\subsection{Women's Bodies Online/on Social Media}

Body image can be understood as psychology of one's body, which is described as "inside perception" (Cash \& Pruzinsky, 1990: p. 53). Alternatively, body image can be defined as "feelings, perceptions, emotions, and beliefs about our bodies, the way we see and feel about our body" (Cash \& Pruzinsky, 1990). In addition, Kyrölä (2016) explained that in order to understand and evaluate an individual's brain it is important for practitioners to determine the connection between brain and bodily experiences. For instance, Lee, Choi, Kim and Han (2014) stated that body image in diverse cultures are found to be influenced both negatively and positively. The body image has a lot to do with appearance which can be perceived differently by different people. These perceptions in this era are influenced by media because it is the widely used mode of communication and interaction. In accordance to Lewallen and Behm-Morawitz (2016), recent developments and trends in internet has influenced the living standards for people. Generally, Lewallen and Behm-Morawitz (2016) stated that in order to remain satisfied with physical appearance, people will have to reject societal ideal and trends of attractiveness. It seems to be impossible for people to reject societal trends and ideals. Thereby, it is important for concerned associates to examine social environments and ensure to present realistic images of individuals. In addition, the study of Toffoletti and Thorpe (2018) explained traditional media images are found to be the key focus of body image because it can lead towards the development of obsession among women. In general, the consumer culture can ensure the development of obsession that requires challenging body images, media displays, images of popular figures and celebrities with beautiful bodies. It should also question the exemplification of good life.

The study of Ariel-Donges, Gordon, Bauman and Perri (2018); Fardouly and Vartanian (2016); Fardouly, Diedrichs, Vartanian and Halliwell (2015) stated that women's body image can play a major role in reflecting cultural obsessions and preoccupations. These obsessions and expectations can influence the way people perceive others. It can increase the chances of individuals being judgmental. Actually, the images and certain physical body shapes and sizes being presented by mass media serve as the guide for people who facilitates them in categorizing people as socially acceptable and unacceptable. The study of Lewallen and Behm-Morawitz (2016) explained that in the case of women, being socially misfit or unacceptable specifically due to body image can turn out to be an ultimate source of low self-esteem. In Western culture, the media representation of women's body image can be demonstrated as thinner and slender bodies. These types of bodies are labelled as attractive that can ensure success as well as youthfulness for them. To be specific, such bodies are also labelled as socially acceptable images that can strengthen self-esteem. On contrary, Chang, Ren and Yang (2018) stated that overweight bodies of women are often associated with 
laziness and dissatisfaction that can lead towards the development of pressure on people.

\subsection{Women's Bodies on Instagram}

Hendrickse et al. (2017) cited that much of the studies and investigations in this era are committed towards explaining the influence of mass media on body image of women. In general, few studies have indicated that social media app that is particularly based on photographs-Instagram is widely used for defining body images and requirements for women. Khamis, Ang and Welling (2017) explained that Instagram is known as an important space where people can investigate and identify body image research. For this platform, everyday individuals have the ability to become an active producer. In certain, the platform is said to be becoming an increasingly popular site via which people set body image goals and targets. On Instagram, the sharing statistics revealed that 60 million photographs are being shared per day. In certain, it is found to be becoming increasing popular. The study of Jones and Heyes (2016); Gill (2016); Baer (2016) supported the fact that the development of social applications like Instagram depends on the development and improvement in visual style of communication. Arguably, it can be assumed that despite billions of individuals participating in online networking, only few people have ensured to stay away from social traps and fake images.

The social media usage is proved to have a negative impact on young women's self-objectification. This objectification can lead towards the development of body image concerns that are related with the increased dissatisfaction and problem for women (Wise, 2018). Moreover, socio-cultural models of body image are accountable of suggesting the ways in which media usage and integration can cause body shaming for women (Azzarito, 2018). On contrary, Slater, Varsani and Diedrichs (2017) stated that an important feature of Instagram is regarded as "fitspiration" images that are sources for fitness and inspiration. These images and photographs are designed to encourage people to exercise and eat healthy. In this manner, objectification in Instagram can be regarded as fruitful for women. In addition, Salter (2016); Bruce (2016); McLean, Paxton and Wertheim (2016) cited that the statistics also unveiled those largest consumers of fitspiration images are young women. Subjectively, it can be assumed that although these images are largely focused on triggering the sense of motivation and encouragement among people to adopt healthy lifestyle, they are also significantly appearance-focused. To be specific, Slater, Varsani and Diedrichs (2017) stated that these images are still primarily contained for women with thin toned body. Apparently, these images are sexually objectifying that can develop unrealistic expectations among people. On contrary, the study of Grogan, Rothery, Cole and Hall (2018) cited that women should exercise and workout with the end goal of becoming fit. These fitspiration ideas should not provoke women to become skinny because it can cause severe health concerns and problems. 
Typically, fitspiration images on Instagram are meant to be developing false body images that can influence their overall abilities and health.

\subsection{Literature Gap and Conclusion}

The considerable review of available literature facilitated in determining that feminism is an important concept that has acquired attention of numerous accredited scholars and practitioners. In fact, on the basis of the above discussion, it can be explained that several existing works conducted on feminism, women's body and post-feminism has transitioned over time. Many accredited scholars and practitioners have extensively studied and examined the importance of feminism. In relation to this concept, some studies have studied post feminism which is also an important subject in this field. However, the body of knowledge of this particular field lacks evidences and information about the recent developments and concerns about women's body image within society. In particular, the available past studies (Retallack et al. 2016; Fardouly et al., 2015) have not assessed this concern in accordance with social sites and platforms like Facebook and Instagram. The evidences available for this investigation lacks information specific to the case of women's body on Instagram and women's body online along with its relation with feminism, women empowerment and post feminism despite that there are a few exceptions. For instance, Brown and Tiggemann (2016) explore influence of Attractive celebrity and peer images on Instagram on women's moods and body image. This lack of information is regarded as a gap in the study which will be addressed with the completion of existing investigation. Apparently, it can be declared that examination about the women's body image and social influences based on feminism can play a major role in contributing towards the body of literature of this specific field. Overall, the identified gap in this study will be addressed through successfully studying and investigating this research.

The overall view of findings demonstrates that in this era, society is responsible of controlling individuals about the way they should show up and shape their bodies. The media acts as a platform for demonstrating how the female body image presentation can influence the perceptions of women's body. Similarly, a significant part of the past investigations have closed the manners by which conventional media has impacted self-perception impression of individuals. Essentially, the reconciliation of media patterns is driving towards the advancement of negative and additionally undesirable self-perceptions among women. Seemingly, it can be accepted that in spite of billions of people taking an interest in web based systems administration, just couple of individuals have guaranteed to avoid social traps and images. As far as fitness is concerned, the media representation depict that women and ladies should exercise and exercise with the ultimate objective of getting to be fit. These fitspiration thoughts ought not to incite ladies to end up thin in light of the fact that it can cause extreme wellbeing concerns and issues. Regularly, fitspiration pictures on Instagram are intended 
to grow false self-perceptions that can impact their general capacities and wellbeing. Overall, it can be concluded and expressed that these pictures on social media are assumed to be influential.

\section{Research Methodology}

\subsection{Introduction}

The methodology chapter forms an important component of overall dissertation. The given chapter facilitates in understanding the methods and techniques being used for collecting desired data and information. The chapter is accountable of explaining characteristics of study specifically in terms of design, approaches and strategies being used. In particular, the discussion in this chapter helps the reader in understanding the methods being used for collecting data and information. Moreover, in accordance with data sets are effectively collected and assembled in this chapter. Subsequent to the explanation of data collection method, data analysis plan is described and illustrated in this chapter.

\subsection{Research Approach}

Selection of suitable research approach is essential for researcher. Usually, qualitative and quantitative are the common research approaches that are being used in research studies. The qualitative approach in a research seeks to explain an issue using experiences and opinions of people (Bryman \& Bell, 2015). It is usually integrated in the form of narration whereas quantitative approach is used in the form of numbers and numerals. The quantitative approach facilitates in conducting an investigation using statistical measures and approximation (Bryman, 2012). As far as existing study is concerned, researcher has used qualitative approach specifically under constructivism. The qualitative approach under constructivism refers to the development of an agenda for reforming and explaining a research problem (Saunders, Lewis, \& Thornhill, 2012). The research has employed qualitative approach from a constructivist standpoint with the end goal of understanding the ways in which Instagram becomes a platform that foregrounds particular body images for women.

Thus, the researcher has integrated qualitative under constructivism because it encourages author in reflecting upon past arguments and opinions relating to women's body image presentation on social media. The qualitative approach is suitable for this study because it facilitates in interpreting emerging texts and image data extracted from Instagram. The qualitative approach is effective in explaining the ways in which past scholars and practitioners understand the problem of the research. It provides a detailed observation and explanation of theories related to research problem and issue. Thereby, integration of qualitative approach for studying females' body image on Instagram is suitable and justifying. The qualitative approach fits in with the nature of the research that is explanatory. It further facilitates in answering the ways in which questions and underlying mechanism that is how females' body image is portrayed and repre- 
sented on Instagram.

\subsection{Research Strategy}

In research, strategy refers to the precise action plan or activity performed by researchers for collecting data (Saunders, Lewis, \& Thornhill, 2012). The selection of suitable research strategy is essential for researcher because it can influence overall authenticity and relevance of findings. In particular, execution of strategy is comprised of actions being taken for aggregating information and data for the completion of an investigation. The commonly used research strategies are interview and questionnaire-based surveys, content analysis, observation and experimentation. The strategy for an investigation is selected based on the nature of issue being selected for investigation (Bryman \& Bell, 2015). The study about female's body image presentation on Instagram has adopted content analysis. In particular, content analysis strategy has been selected for this study because it is a method used for studying documents and arguments of accredited scholars and academicians. The selection of content analysis strategy for existing study is suitable because it assists in systematically evaluating and describing text based information about a selected problem. Thereby, content analysis is effective in analyzing content relating to women's body image presentation on Instagram. In likewise manner, integration of content analysis strategy for existing study is suitable because it attempts to summarize different forms of content available for the study. With content analysis, researcher attempts to evaluate images, visuals, trends and hashtags related to women's body image presentation on Instagram. Another reason behind using content analysis strategy for existing study is that it facilitates in examining different patterns of social experiences of people about the selected problem. It attempts to indicate presence and existence of interesting and meaningful content.

\subsection{Data Collection}

The data collection process refers to the systematic planning followed by the researcher for collecting desired data and outcomes (Bryman, 2012). Moreover, data collection can be evaluated as the process of gathering as well as measuring information relating to variables of interests. The explanation of data collection process is essential for researcher because it strengthens authenticity and relevance of obtained findings (Saunders, Lewis, \& Thornhill, 2012). Prior to the explanation of data collecting process, it is mandatory for researcher to examine sources being used for collecting data. In usual cases, social science based studies employ primary or secondary data collecting sources. Primary sources are accountable of offering updated and unused information which can be collected in the form of observations and responses of participants. On contrary, secondary sources of data collection offer past studies and publications. The secondary sources offer already published information and data for the study (Collis \& Hussey, 2013). 
In the research, secondary data collection is used. Precisely, it can be stated that secondary sources are suitable for this study because it facilitates in evaluating recent trends and Instagram fashions. The study is likely to extract data from past studies, blogs and articles. Evidently, data and information used in this study is already published and interpreted.

\subsection{Data Set}

The study is further likely to review comments and preferences of people on Instagram for women's body image. The datasets further include hashtags (\#) and @ user tags for determining the accounts focusing on presenting women's body images. Purposively, a small sample of popular hash-tags was selected based on the top listed trends. The used hashtags are like \#Stayfit, \#Instafit, \#BeachBodyGoals, \#EatClean and \#Bodytransformation. These hashtags for the existing study are selected due to their relevance with body image of women. The ideas extracted from this study are also obtained via keywords like body and size. The selection of pictures facilitated in critically evaluating body image trends being shared and celebrated online. The top six photographs identified under each of the hashtags were selected and they were screenshot over a period between January to March 2018. The collected datasets are categorized in the form of screenshots. A total of 100 photographs are analysed as well as the comments on the images.

In addition, the “@” tag is used for exploring specific accounts to help investigate into how women are disseminating the body image on Instagram. The research purposively select the 5 of the most popular female fitness accounts on Instagram (see Table 1). The researcher turns to Harper's Bazaar (Harpersbazaar.com) for learning the most popular female fitness accounts. During the period, the researcher collects a total of 40 photographs. Both images and comments are analyzed.

\subsection{Data Analysis}

In order to obtain authentic findings and outcomes for research, it is imperative for researcher to adopt suitable data analysis plan. In certain, qualitative can be analyzed using content analysis/coding process, thematic and ethnographic content analysis. On contrary, quantitative data can be evaluated using statistical software like SPSS or excel sheets (Bryman, 2012). As far as existing study is concerned, it has been declared that researcher has applied qualitative approach under constructivism. The integration of this approach encourages author to select content analysis grid and coding processes. The integration of this method is likely to facilitate in collecting and evaluating collected data and obtaining relevant outcomes. The coding processes and content analysis grid facilitates in organizing images selected for the study. The grids and coding processes assist in evaluating trends associated with the representation of women's body on Instagram. The selection of content analysis grid and coding is suitable for the existing study since it is accountable of producing themes for data interpretation. 
Table 1. Datasets.

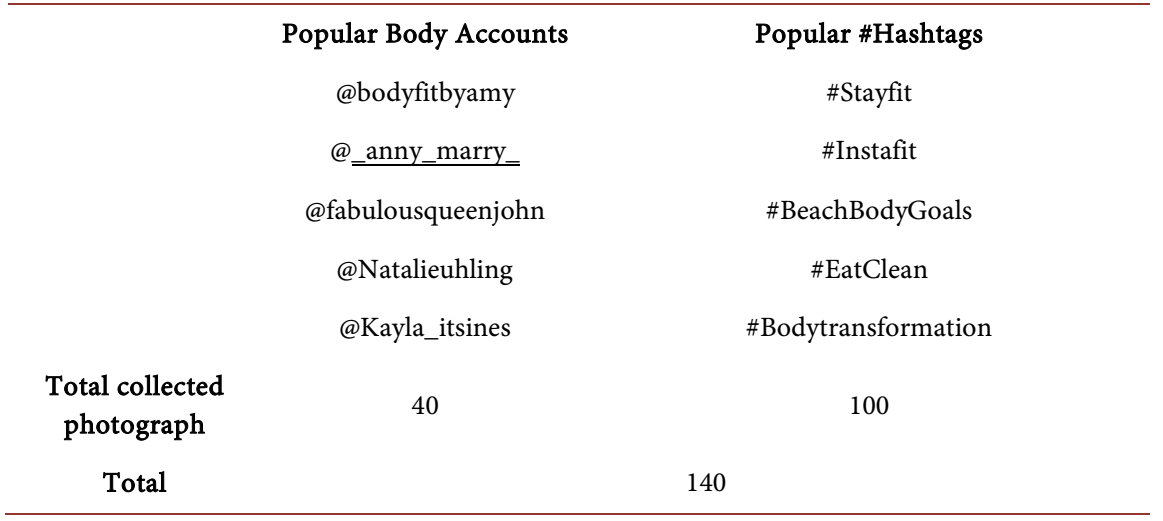

\subsection{Limitations}

The limitations in research are called uncontrollable influences that occur throughout the completion of an investigation. The limitations are common in research; thereby, it is mandatory for researcher to address them in dissertation (Bryman, 2012). The limitations for the existing study are listed down as 1) The study is likely to face a limitation in terms of data collecting measures being used. The existing study has employed qualitative data only which can occur as a limitation for this study. The limitation is expected to influence the scope and applicability of findings. 2) The study is also likely to encounter lack of longitudinal effects. The research is cross-sectional. Thus, it may fail to reflect the real-life situations as time goes on as females may have their attitude towards their body image changed, or use Instagram differently.

\subsection{Conclusion}

The overall view of chapter facilitates in understanding that in order to study and evaluate the ways in which female body image is presented and interpreted on Instagram researcher has developed and followed a systematic methodology. To be specific, the study has employed content analysis strategy. The approach being employed is qualitative under constructivism. The data is collected in the form of specific datasets. These datasets are in the form of hashtags and popular accounts. The collected data is analyzed using content analysis grids and coding processes.

\section{Findings and Discussion}

\subsection{Introduction}

The data analysis chapter is presenting findings and outcomes. In certain, chapter facilitates in evaluating collected data and information for the study. The discussion in this chapter interprets related codes and themes so as to illustrate key findings of the research. Subsequent to the illustration of research findings, author intends to provide a detailed discussion of findings. The discussion of findings attempts to compare results with findings in previous research. Appar- 
ently, the discussion of findings facilitate in evaluating relevance and usage of academic literature relating to research outcomes against developed objective. Moreover, discussion section of the research is accountable of speculating and identifying differences and similarities between findings and established literature. Precisely, chapter facilitates in evaluating whether obtained findings are effective in answering research questions or not. Lastly, a comprehensive conclusion highlighting key findings is also added in this chapter.

\subsection{Female Body Image Represented on Instagram}

Body image highlighting sexuality

Analysis of dataset reveals that the women's body image is represented in a way of highlighting "sexuality" and attractiveness. The pictures reviewed on Instagram depicted that perfect body image representations and visuals include comments like "Sexy" and "Body goals". These comments are accountable of giving rise to certain societal trends. Instagram users are playing a major role in forming ideas around women's body. The body positive account holders are found to be having different reasons and concerns for representing themselves on platform. These pictures are accountable of contributing towards the development of motivation and interest both. Moreover, the review of selected accounts and pictures depict that the photographs being shared online tend to mainstream images specifically within traditional media. The development of Instagram pictures tends to attract attention and perception. Attractive public accounts get millions of followers that encourage in developing and giving rise to trends. One of the commonly known public accounts being reviewed in the existing study is of @Kayla_itsines. Figure 1 comes from @KayLai-ItSies.

The account tends to sell bikini body goals and guide for young females. The display pictures are widely liked and shared by followers making her Instagram star and influencer. These body images display attractiveness, with the sense of perfectness. For example, Figure 2.

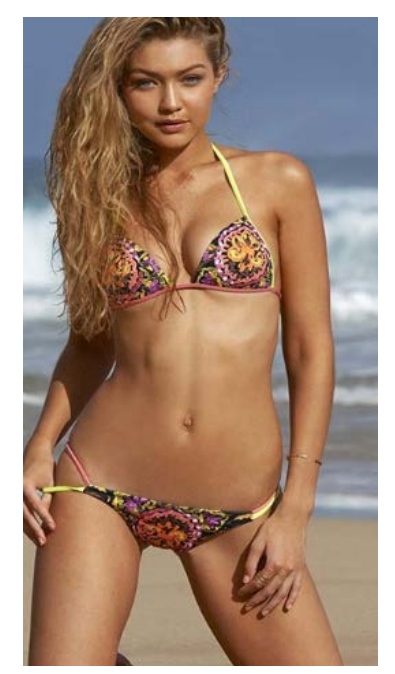

Figure 1. Body image. 


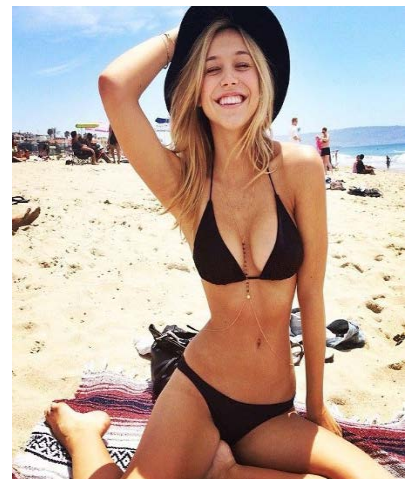

Figure 2. Body image.

Most of such pictures add captions like "goals" and "Perfection". These captions tend to develop an expectation and desire among people. The constant reminder of such comments via hashtags tends to influence perception and opinions of people. Moreover, from the given images, it has been found that some pictures on Instagram representing transformation by adding before and after pictures tend to trigger the sense of motivation among people. Some of such body image pictures are considered as inspirational.

\section{Body image addressing business purpose}

In addition, it was found that Instagram accounts are also used for business purposes as well. There are many public accounts that promote healthy eating brands and cardio machines. For instance, @bodyfitbyamy in collaboration with \#Bowflex launched cardio machine. @bodyfitbyamy is known for presenting women body goals which make it effective for women to get in shape. To be specific, the findings facilitate in determining that the influencers are paid for displaying body images and advertising resources and different products. Typically, Instagram is widely used as a platform that can change the way people perceive their bodies. It is effective in challenging normative believes and perception of people about the way they should appear. For instance, Figure 3 demonstrates a woman with fitness product $\mathrm{C}$, with a big smile.

The image creates the impression that the product is satisfactory. Generally, the findings demonstrated that women are known as great influencers online because they have the ability to stay active and produce desired pictures and images. In fact, women are easily able to get desired visual communication patterns that facilitates in promoting themselves as well as empowering other women. The hashtag trend of social media is effective in generating trends because photographs mostly use ideas pertaining around bodies. Consequently, these images make sure that they are generating positive image and reputation for people.

Body image suggesting ideal body

Moreover, it was found that the female body image was represented in a way for suggesting the ideal body. The ideas pertaining to body images are able to control selected values of society. Instagram is playing an important role in influencing body image of women because people carefully select pictures and content to put online. The added hashtags give away certain amount of idea 
about the picture being shared. In such cases, people are not necessarily being authentic which triggers the sense of unrealistic body image expectations among them. For instance, Figure 4 influencers and celebrities posting images with \#BeachBodyGoalshashtag tends to cultivate unrealistic body expectations among women.

It has been evaluated that women in particular are not directly controlled about the content they upload and present themselves in photographs on Instagram. Certainly, it has been found that Instagram pictures and images are not always authentic which can deceive people. In many images, the focus subject remains the concern for people. After constantly looking at perfect pictures, women end up assuming and expecting differently. The appreciating comments on women's body images and pictures can influence self-esteem and perception of others. In the case when women do not fit in these expectations of having specific body, they end up spoiling health. For instance, majority of Instagram body image pictures present women in an "hour glass shape" which sets unrealistic expectations among people. Relatively, general view of selected images depicts that the developments of specific body images are accountable of affecting personal reputation and perception among people. Up to some extent, body images are effective in encouraging women to do workout and adopt a healthier lifestyle. However, it has been analyzed that these photos can also turn out to be deceiving which causes discouragement among them. Substantially, body structures of people are different from each other. The feminine look body images can influence self-esteem and confidence of females.

\section{Other body images}

While most of body images suggest that women should be slim and sexy, some images are challenging the majority. Some photos show women are lifting heavy weights or doing something adventure. For instance, Figure 5 demonstrates that a woman is running over fires. This is quite contrary to the ideal image of women imposed by the society. Another example can be found at @Honorcurves. Figure 6 demonstrates a woman wearing a bikini at a larger size, and communicating the significance of being happy without worry about being ideal of perfect in body.

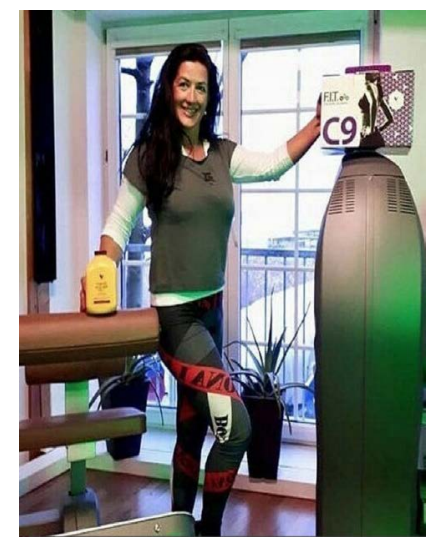

Figure 3. Body image linking with fitness product. 


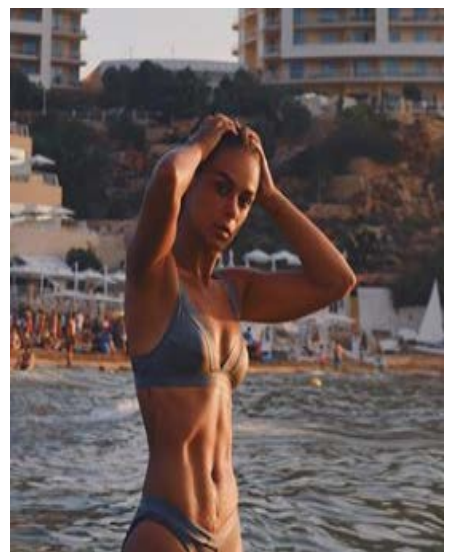

Figure 4. Body image found with \#BeachBodyGoalshashtag.

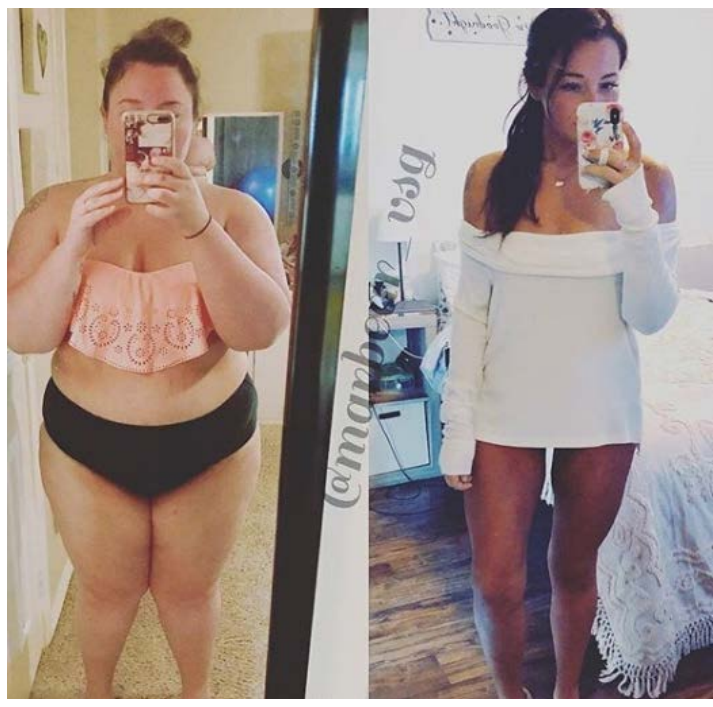

Figure 5. Body image.

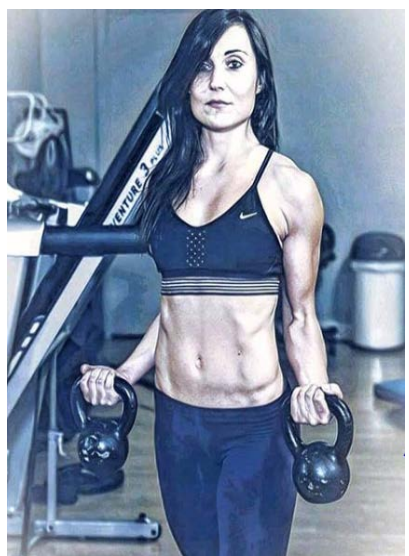

Figure 6. Edited images on instagram.

\subsection{Responses to Women Bodies Image on Instagram}

Reviewing the data about the viewers' comments on the body image communicated on Instagram, it is found that among the 140 collected photographs, there 
is an average of 30 comments for each photograph, and an average of 2000 likes for each photograph. The most mentioned comments are "nice", "gorgeous", "inspiring" and others.

\section{Comments showing admiration and appreciation of the ideal body}

The findings highlight that most viewers have positive attitude towards the body image shared on Instagram. They appreciate the wonderful body image, and some are startled by the transformation in body image, and admire the transformation. For instance, Figure 5 to Figure 6 demonstrate the dramatic change of body image. This triggers appreciation and admiration. Considering responses to women body images on Instagram, it can be stated that body satisfaction is an important concern for individuals. Apparently, state body satisfaction is found to be measuring before and after views of people. Based on comments on transformation, it is found that transformation trends are likely to influence the motivation and encouragement among people about being healthy. The key responses to women body image on Instagram are significantly related with body satisfaction trends. The lack of body satisfaction increases among women due to unrealistic postings and pictures. Relatively, findings depicted that body appreciation is found to be an important measure of satisfaction. The absence of satisfaction is usually the result of lack of body appreciation. The comments on transformation images on Instagram were like "GIRL YOU INSPIRE ME SO MUCH! I'm so happy we found you" and "You look bloody fantastic girl". These comments depict significant consideration and acceptance of female body images on Instagram.

\section{Comments indicating dissatisfaction with themselves}

Moreover, the comments suggest that the images on Instagram trigger self-dissatisfaction. The ideal body images trigger the sense of dissatisfaction about body image among the viewers. For instance, the comments on above given Instagram picture was like "Wow, awesome transformation! Check out mine" and "I gotta do it too" depicts the interest of women in transforming their bodies.For example, Figure 5, it has been further noted that the self-worth and importance in influenced by the illustration of body image pictures.

The response of women's body image on Instagram can be addressed specifically in terms of increased pressures. To be specific, the pressures on women to achieve perfect body are found to be getting worse due to the increased development of internet. For instance, women body image disgusts thigh touching or rubbing together. Considerably, these pictures on Instagram tend to encourage thigh gap fixation. These desires can cause several unhealthy results for women. Majority of responses of women on body image on Instagram are influenced by fitspiration trends. However, it can cause several problems and unrealistic expectations among people. The general view of selected images and accounts facilitate in declaring that Instagram is widely used among people because it triggers the sense of empowerment among people. Many comments on perfect body pictures tend to encourage women to get in shape. In fact, comments like gor- 
geous, perfect and stunning makes women female empowered because it triggers the sense of being an influence for people. This empowerment over others tends to make people feel good about them. Apparently, it has been noted that Instagram is primarily viewed as visual that possess few limitations in photographs. Instagram allows people to share pictures, upload videos and visuals which empower all users. Pictures and visuals shared on Instagram tend to influence psychological perceptions and opinions of people about body image. In certain, it is clear that body image is strongly linked with optics that makes Instagram as an importance socializing and communicating space for women. The analysis of accounts focused on representing women's body facilitate in analyzing that there are several different ideas and expectations associated with bodies of women. In fact, Instagram is found to be primarily used for producing same ideas conceiving women's bodies as ideal. Considering images shared on Instagram, it can be assumed that in many cases, female body image representing accounts on Instagram tend to empower women that facilitates in creating change. Arguably, female body image representation of Instagram can be categorized in specific themes. These themes are categorized as body images, resistance, motivation, before and after comparison pictures and posing staged advertisements.

Comments indicating self-objection

While social media is widely used and encouraged among young people, execution of social media possesses several negative impacts on human life. Women's responses to Instagram images are determined to be the source of self-objectification. The above transformation images depict deceiving because they are edited. Forexample, Figure 6. The body image expectations can trigger the sense of complex among people. The pressure of body image responses can cause women to become trapped by eating disorders. For instance, comments like "Need to start workout Now" or "I should be doing it more" demonstrates the dissatisfaction among women.

These eating disorders can lead towards the development of health issues and problems. The responses among women about body images are found to be associated with socio-cultural models. Instagram is found to be influenced by body images and self-objectification trends. It is widely known for shaping women's responses to body expectations and requirements. After seeing at perfect pictures, women tend to opt for surgeries and deadly diets.

Generally, the findings facilitate evaluating that Instagram is significantly influencing because it is known as an image-based social media platform with over 80 million images being uploaded on it every day. Instagram is deceiving because there are several editors and filters available in this application. People mostly edit and enhance image features using different filters being provided by Instagram. The edited pictures make women feel uncomfortable and uneasy about their bodies. Thereby, it can be stated that most of the responses shared by women against Instagram pictures lead towards the reduction in body satisfaction. The body image and satisfaction are handy with each other. 
Instagram is effective because 80 million images uploaded to Instagram every day provide users with numerous opportunities to compare their appearances to others. Instagram post are effective in promoting societal beauty standards and ideals that result in judgements. These judgements tend to influence overall body acceptance abilities among women. Apparently, women trapped with unrealistic body expectations feel less attractive than others. Due to constant comparison, people end up trapped in body objectification.

\subsection{Discussion}

\subsubsection{How Women's Body Images Are Presented Instagram}

The findings display female body image is presented and interpreted on Instagram. Among the 140 selected photographs, it is found that the presentation of body image emphasizes attractiveness, sexualities, and perfectness dominate the almost 120 photographs. In addition, the body image also communicates in a way of advertising a specific product and promoting an ideal body. the portrayal is similar to the traditional media's presenting image of beautiful bodies. Moreover, the photographs suggest women in minimal clothing such as bikinis. Their bottoms are exposed, suggesting sexy images. These are congruent with the traditional female image that is expected by the society.

In Western culture, the media portrayal of ladies' self-perception can be shown as more slender and thin bodies. These sorts of bodies are marked as alluring that can guarantee accomplishment and in addition energy for them. In particular, such bodies are likewise marked as socially worthy pictures that can fortify confidence. Chang, Ren and Yang (2018) expressed that overweight assemblages of ladies are regularly connected with apathy and disappointment that can lead towards the advancement of pressure on individuals. Lewallen and Behm-Morawitz (2016) noticed that people's social surroundings are observed to be related with their associations with individuals. In certain, it contributes towards the general improvement of thoughts and suppositions that can commend principles and necessities of self-perception. The investigation of Lee, Lee, Choi, Kim and Han (2014) clarified that self-perception rivalry is high among ladies since social destinations are assuming a noteworthy part in celebrating the guidelines and prerequisites for them. The results indicate that the traditional images and norms imposed on women remain overall.

\subsubsection{How Body Image Presentation on Instagram Influences Women}

The comments reveal to some extent the influence of body image presentation on Instagram on women.

\section{Arousing consciousness and inspiration}

First, the comments suggest that the body image on Instagram can yield consciousness among women that can facilitate them in creating change. Some of the responses being shared by women for Instagram images are evaluated as empowerment. Apparently, empowerment does not occur with consumptions; in fact, it relates with the development of awareness among women. Typically, 
Instagram pictures shared by @_anny_marry_can lead towards the development of sexiest assumptions about women. These assumptions cause several expectations among people regarding the body image and structure of women. Instagram images are widely known for presenting and portraying women's body in a way that it attracts viewers.

Majority of the comments on images said "Inspirational", "Amazing body", "Gorgeous". These comments encourage other female followers to adopt similar lifestyle. It has been noted that women get highly concerned about getting suitable body images and reputation. As far as media is concerned, it can be assumed that it plays an important role in advancing sexual objectification which specifically facilitates people in addressing beauty standards. Feminism is set to eliminate pressure from women because it can help in gaining equal rights. The media portrayal of women has settled high standards for women. Thereby, women require relief from such unrealistic expectations and intentions. Overall, it can be interpreted that feministic approaches play a major role in advancing security and support for women. The approach can play a major role in empowering women without adding pressure or influence. Relatively, Instagram pictures and body image of women is so conventional that it can lead towards the development of health issues for them. Thus, feministic approaches and movements are required.

In general, obtained findings and outcomes of this study demonstrated that female self-perception portrayal of Instagram can be viewed as an important concern. These subjects are arranged as self-perceptions, opposition, inspiration, when Instagram pictures are displayed. The appealing self-perceptions of females are broadly considered and seen as great. The vast majority of such pictures include subtitles like sexy and flawlessness. The obtained findings and results are aligned with the study of Lee, Lee, Choi, Kim and Han (2014) who explained that the pressure of self-perception reactions can make females end up caught by dietary issues. The desire to get idealized body can lead towards the development of unrealistic planning like diet and workout which can turn out to be harmful for their health. These dietary issues can lead towards the improvement of medical problems and issues. The reactions among females about self-perceptions are observed to be related with socio-social models. Instagram is observed to be affected by self-perceptions and self-externalization patterns. It is broadly known for influencing females' reactions to body desires and necessities. Subsequent to seeing at idealize pictures, females have a tendency to select medical procedures and lethal eating practices.

\section{Triggering self- self-objectification}

The analysis of Instagram images further demonstrated that traditional media is primarily known for featuring images of celebrities and models to influence women. Women often compare themselves and end being dissatisfied. Instagram features can somehow be used for fitspiration. The fitspiration videos, accounts and pictures tend to promote healthy living standards among people. The 
development of fitspiration can cause effective benefits for people. However, fitspiration videos are accountable of guiding and motivating people. On contrary, some cases of fitspiration can cause unrealistic expectations among people. Due to these cases, women get involved with unhealthy diet plans and workout plan. The trend of fitspiration and self-compassion are interlinked with each other. Instagram images are effective in strengthening body image reputation and perception among women.

The comments revealed that the viewers are much more likely to compare their own images with the images communicated on Instagram, showing admiration and dissatisfaction with their own bodies. For instance, one comment reveals that she is not satisfied with her body after reviewing the ideal body image on Instagram. The findings are congruent with the established literature concern the self-objectification influenced by social media. The idea of objectification in feminism is regarded as a central notion. It can be defined as an important problem being faced by people living across the globe. It has been noted that objectification can be identified in terms of reduction to body. It treats people in the form of identified body structures and images. Many themes have emerged from data analysis depicting that sexual objectification is strongly associated with the objectification of influential contemporary images. The development of sexual objectification can strongly relate with feminist theories. The pictures and images available online are bound to relate with the development of women objectification (Azzarito, 2018; Slater et al., 2017). The images on Instagram facilitate in determining that the objectification is accountable of causing several health issues and problems for women's development. Instagram pictures are accountable of influencing women's image and reputation. It also relates with the development of unrealistic expectations that can cause severe problems and issues for their health. The idea of women empowerment is significantly influenced by Instagram pictures and images. The development of images on Instagram can affect perception of people specifically regarding their bodies. The media patterns are effective in shaping norms and perceptions about the people. In particular, objectification of women is strongly related with the development of Instagram based images and pictures. The feministic approaches are accountable of eliminating issues like women objectification. Instagram pictures are accountable of shaping perception and opinions of people about their bodies.

Stimulating pursuit for a non-traditional image

While there are only few comments suggesting resistance against the social norms of body images, these images may inspire adopting a new perspective to perceive and expect the body image. Feminism is an important idea being discussed in association with women across the globe. Apparently, feminism can be explained and viewed as a range of ideologies that lead towards the development of different movements and an ideology that struggles to get equal rights for women (Tiggemann \& Zaccardo, 2015; Modleski, 2014). The establishment of feminism approaches are accountable of acquiring personal, political and social 
equality for people belonging from different genders. The equality trends are effective in maintaining women's rights and interest. The feministic approaches are required to eliminate women objectification from society. One of the common examples being discussed in this domain relates with the development of Instagram body images of women. The portrayal of women online plays a major role in women objectification. The development of women objectification is accountable of affecting self-esteem, performance, health and stability of women.

However, the comments suggest distortion. There is a danger that the communicating too much the ideal body may result in women's suspecting themselves. The female body presentation online encounters several different challenges and problems. The developments of Instagram pictures are accountable of increasing stress and pressure on people. As revealed by comments on the images on Instagram, many viewers have expressed dissatisfaction with their own bodies, and some even hated their current bodies. In order to eliminate the trends of social media like Instagram, it is important for concerned associates to relate with feministic approaches. The advancements of trends can facilitate in strengthening image and reputation among people. It considers the ways in which people triggers the sense of accomplishment and advancement. The findings are determined to be in line with the literature presented by Lee et al. (2014) who expressed that self-perception influence is resolved to be an essential and viable issue among females. Because of these distorted or edited pictures and photos, weight concerns are likely to relate with females. The development of unrealistic images can contribute towards the development of societal standards. The study completed by Feltman and Szymanski (2018) also supported the fact that majority of females in society trust that that they are overweight and are worried about their negative appearances. These appearances can relate with the advancement of issues and problem among females. Typically, negative self-perceptions and drive of slimness are driving towards the advancement of dietary problem among women.

\section{Conclusion}

\section{Summary of Findings}

The review of data analysis and discussion facilitates in claiming that researcher has accomplished developed objectives of this study. The obtained findings in accordance with objectives are presented as follows:

How women's body images are presented on Instagram

The findings reveal that how women's body image is communicated on Instagram. It is found that body images presented in Instagram highlight sexuality and attractiveness by emphasizing slim body, long hair, with fewer clothing. The photographs review on Instagram depicted that perfect body image representations and visuals. In addition, the body images on Instagram are presented in a way for communicating business purposes. Many public accounts are found to promote healthy eating brands and cardio machines. Besides, it is found that the 
female body image was represented in a way for suggesting the ideal body. While the majority of body images communicate that women should be slim and sexy, some images are challenging the majority by creating totally different images, such as women's lifting heavy weights or doing something adventure and wearing a bikini at a larger size. Overall, among the 140 selected photographs, 120 photographs communicate attractiveness, sexualities and perfectness. The portrayal is congruent with the traditional media's presenting image of beautiful bodies.

How viewers respond to women's body images communicated on Instagram

It is found that most viewers positively respond to body image photographs in Instagram. Most of them appreciate wonderful and ideal body image, and some are startled by the transformation in body image, and admire the transformation. In addition, it is found that many comments show that the viewers are dissatisfied with themselves after comparing their body images with ideal body images. Third, accompanying the dissatisfaction with their own body, the comments reveal self-objectification. The pressure of body image responses can cause women to question their own images.

How women's body images communicated on Instagram influence the view$\underline{\text { ers }}$

Drawing from comments, it is found that the women's body images communicated on Instagram influence the viewers significantly in the following aspects: 1) It is found that women's body image communicated on Instagram can arouse consciousness and inspiration so as to encourage them to make changes. 2) It is found that the women's body images communicated on Instagram trigger self-objectification. The viewers are much more likely to compare their own images with the images communicated on Instagram, showing admiration and dissatisfaction with their own bodies. The findings are congruent with the established literature concerning the self-objectification influenced by social media. 3) While the body images resisting against the social norms of body images are less on Instagram, these images may inspire use of a new perspective to perceive and expect the body image. This is congruent with feminism's advocating sex equalities. The findings can enrich the established literature by providing lens that the future research can focus on exploring the influence of body image on Instagram through critically reviewing the comments.

\section{Conflicts of Interest}

The author declares no conflicts of interest regarding the publication of this paper.

\section{References}

Andsager, J. (2014). Research Directions in Social Media and Body Image. Sex Roles, 71, 407-413. https://doi.org/10.1007/s11199-014-0430-4

Ariel-Donges, A. H., Gordon, E. L., Bauman, V., \& Perri, M. G. (2018). Does Yoga Help 
College-Aged Women with Body-Image Dissatisfaction Feel Better about Their Bodies? Sex Roles, 1-11.

Azzarito, L. (2018). Re-Focusing the Image of the "Superwoman" with "No Colour": "Writing Back to the Centre" from a Globalised View. In New Sporting Femininities (pp. 135-157). Cham: Palgrave Macmillan.

https://doi.org/10.1007/978-3-319-72481-2_7

Baer, H. (2016). Redoing Feminism: Digital Activism, Body Politics, and Neoliberalism. Feminist Media Studies, 16, 17-34. https://doi.org/10.1080/14680777.2015.1093070

Banet-Weiser, S. (2018). Postfeminism and Popular Feminism. Feminist Media Histories, 4, 152-156. https://doi.org/10.1525/fmh.2018.4.2.152

Brown, Z., \& Tiggemann, M. (2016). Attractive Celebrity and Peer Images on Instagram: Effect on Women's Mood and Body Image. Body Image, 19, 37-43. https://doi.org/10.1016/j.bodyim.2016.08.007

Brown, T. (2017). Examining the Influence of Social Media on Body Image: Miss Perfection, a Misperception (Doctoral Dissertation, Colorado State University. Libraries).

Bruce, T. (2016). New Rules for New Times: Sportswomen and Media Representation in the Third Wave. Sex Roles, 74, 361-376. https://doi.org/10.1007/s11199-015-0497-6

Bryman, A. (2012). Social Research Methods. Oxford: Oxford University Press.

Bryman, A., \& Bell, E. (2015). Business Research Methods. Oxford: Oxford University Press.

Cameron, E., Ward, P., Mandville-Anstey, S. A., \& Coombs, A. (2018). The Female Aging Body: A Systematic Review of Female Perspectives on Aging, Health, and Body Image. Journal of Women \& Aging, 1-15. https://doi.org/10.1080/08952841.2018.1449586

Cash, T. F., \& Pruzinsky, T. E. (1990). Body Images: Development, Deviance, and Change. New York: Guilford Press.

Ceulemans, M., \& Fauconnier, G. (2015). MASS MEDIA: The Image, Role, and Social Conditions of Women: A Collection and Analysis of Research Materials, UNESCO. International Women Online Journal of Distance Education, 4, No. 2.

Chang, J., Ren, H., \& Yang, Q. (2018). A Virtual Gender Asylum? The Social Media Profile Picture, Young Chinese Women's Self-Empowerment, and the Emergence of a Chinese Digital Feminism. International Journal of Cultural Studies, 21, 325-340. https://doi.org/10.1177/1367877916682085

Collis, J., \& Hussey, R. (2013). Business Research: A Practical Guide for Undergraduate and Postgraduate Students. London: Palgrave Macmillan.

Coppock, V., Haydon, D., \& Richter, I. (2014). The Illusions of Post-Feminism: New Women, Old Myths. Abingdon-on-Thames: Routledge.

Fardouly, J., \& Vartanian, L. R. (2016). Social Media and Body Image Concerns: Current Research and Future Directions. Current Opinion in Psychology, 9, 1-5. https://doi.org/10.1016/j.copsyc.2015.09.005

Fardouly, J., Diedrichs, P. C., Vartanian, L. R., \& Halliwell, E. (2015). Social Comparisons on Social Media: The Impact of Facebook on Young Women's Body Image Concerns and Mood. Body Image, 13, 38-45. https://doi.org/10.1016/j.bodyim.2014.12.002

Fardouly, J., Willburger, B. K., \& Vartanian, L. R. (2018). Instagram Use and Young Women's Body Image Concerns and Self-Objectification: Testing Mediational Pathways. New Media \& Society, 20, 1380-1395. https://doi.org/10.1177/1461444817694499

Feltman, C. E., \& Szymanski, D. M. (2018). Instagram Use and Self-Objectification: The Roles of Internalization, Comparison, Appearance Commentary, and Feminism. Sex Roles, 78, 311-324. https://doi.org/10.1007/s11199-017-0796-1 
Gill, R. (2016). Post-Postfeminism: New Feminist Visibilities in Postfeminist Times. Feminist Media Studies, 16, 610-630. https://doi.org/10.1080/14680777.2016.1193293

Grogan, S. (2016). Body Image: Understanding Body Dissatisfaction in Men, Women and Children. Abingdon-on-Thames: Routledge. https://doi.org/10.4324/9781315681528

Grogan, S., Rothery, L., Cole, J., \& Hall, M. (2018). Posting Selfies and Body Image in Young Adult Women: The Selfie Paradox. The Journal of Social Media in Society, 7, 15-36.

Halliwell, E. (2015). Future Directions for Positive Body Image Research. Body Image, No. 14, 177-189. https://doi.org/10.1016/j.bodyim.2015.03.003

Hempel, J. (2014). Instagram Is Getting So Good at News, It Should Scare Twitter. Wired.

Hendrickse, J., Arpan, L. M., Clayton, R. B., \& Ridgway, J. L. (2017). Instagram and College Women's Body Image: Investigating the Roles of Appearance-Related Comparisons and Intrasexual Competition. Computers in Human Behavior, 74, 92-100. https://doi.org/10.1016/j.chb.2017.04.027

Jones, M., \& Heyes, C. J. (2016). Cosmetic Surgery in the Age of Gender. In Cosmetic Surgery (pp. 17-34). Abingdon-on-Thames: Routledge. https://doi.org/10.4324/9781315574387

Khamis, S., Ang, L., \& Welling, R. (2017). Self-Branding, “Micro-Celebrity” and the Rise of Social Media Influencers. Celebrity Studies, 8, 191-208.

https://doi.org/10.1080/19392397.2016.1218292

Kinsaul, J. A., Curtin, L., Bazzini, D., \& Martz, D. (2014). Empowerment, Feminism, and Self-Efficacy: Relationships to Body Image and Disordered Eating. Body Image, 11, 63-67. https://doi.org/10.1016/j.bodyim.2013.08.001

Kyrölä, K. (2016). The Weight of Images: Affect, Body Image and Fat in the Media. Abingdon-on-Thames: Routledge. https://doi.org/10.4324/9781315551463

Lee, H. R., Lee, H. E., Choi, J., Kim, J. H., \& Han, H. L. (2014). Social Media Use, Body Image, and Psychological Well-Being: A Cross-Cultural Comparison of Korea and the United States. Journal of Health Communication, 19, 1343-1358. https://doi.org/10.1080/10810730.2014.904022

Lewallen, J., \& Behm-Morawitz, E. (2016). Pinterest or Thinterest: Social Comparison and Body Image on Social Media. Social Media + Society, 2, 5-27. https://doi.org/10.1177/2056305116640559

Marwick, A. E. (2010). Status Update: Celebrity, Publicity and Self-Branding in Web 2.0. Doctoral Dissertation, New York: New York University.

Mabe, A. G., Forney, K. J., \& Keel, P. K. (2014). Do You "Like" My Photo? Facebook Use Maintains Eating Disorder Risk. International Journal of Eating Disorders, 47, 516-523. https://doi.org/10.1002/eat.22254

McLean, S. A., Paxton, S. J., \& Wertheim, E. H. (2016). The Role of Media Literacy in Body Dissatisfaction and Disordered Eating: A Systematic Review. Body Image, 19, 9-23. https://doi.org/10.1016/j.bodyim.2016.08.002

Modleski, T. (2014). Feminism without Women: Culture and Criticism in a "Postfeminist" Age. Abingdon-on-Thames: Routledge.

Peterson, R. D., Grippo, K. P., \& Tantleff-Dunn, S. (2008). Empowerment and Powerlessness: A Closer Look at the Relationship between Feminism, Body Image and Eating Disturbance. Sex Roles, 58, 639-648. https://doi.org/10.1007/s11199-007-9377-Z

Retallack, H., Ringrose, J., \& Lawrence, E. (2016). “Fuck Your Body Image”: Teen Girls’ Twitter and Instagram Feminism in and around School. In Learning Bodies (pp. 85-103). Singapore: Springer. 
Salter, M. (2016). Privates in the Online Public: Sex (Ting) and Reputation on Social Media. New Media \& Society, 18, 2723-2739.

Saunders, M., Lewis, P., \& Thornhill, A. (2012). Research Methods for Business Students. Pearson.

Slater, A., Varsani, N., \& Diedrichs, P. C. (2017). \# fitspo or\# loveyourself? The Impact of Fitspiration and Self-Compassion Instagram Images on Women's Body Image, Self-Compassion, and Mood. Body Image, 22, 87-96.

Tiggemann, M., \& Zaccardo, M. (2015). "Exercise to Be Fit, Not Skinny": The Effect of Fitspiration Imagery on Women's Body Image. Body Image, 15, 61-67.

Tiidenberg, K., \& Gómez-Cruz, E. (2015). Selfies, Image and the Re-Making of the Body. Body \& Society, 21, 77-102.

Toffoletti, K., \& Thorpe, H. (2018). Female Athletes' Self-Representation on Social Media: A Feminist Analysis of Neoliberal Marketing Strategies in "Economies of Visibility". Feminism \& Psychology, 28, 11-31.

Weidhase, N. (2015). "Beyoncé Feminism" and the Contestation of the Black Feminist Body. Celebrity Studies, 6, 128-131.

Wise, J. (2018). The Curvy Girl Handbook vs. the Industry: A Unique Body Image in a One-Track Business. 